\title{
Localization of Acoustic Signals Used in Sound Emitters at Pedestrian Crosswalks
}

\author{
M. NiewiarowiCz ${ }^{a, *}$ AND A. FURmann ${ }^{b}$ \\ ${ }^{a}$ Department of Otolaryngology, Poznań University of Medical Sciences \\ Przybyszewskiego 49, 60-355 Poznań, Poland \\ ${ }^{b}$ Institute of Acoustics, Adam Mickiewicz University, Umultowska 85, 61-614 Poznań, Poland
}

\begin{abstract}
This study aimed at determining an optimal acoustic signal, which could be used in sound emitters at blind and visually impaired enabled pedestrian crosswalks. Two signals were identified from among groups of tested signals on the basis of psychoacoustic tests. These two signals met the following standard requirements: TR signal - a signal with a triangular temporal envelope and a sinusoidal carrier and RC signal - a signal with a rectangular temporal envelope and a rectangular carrier, both with a basic frequency of $880 \mathrm{~Hz}$, repeated periodically with a frequency of $5 \mathrm{~Hz}$. The ability to localize was tested by a modified method of angle of directional hearing acuity in which the two alternative forced choice adaptation procedure was used. The test signals were emitted against the background of traffic noise and the ratio of the useful signal (65 dB SPL) to noise $(75 \mathrm{~dB}$ SPL) was $(-10 \mathrm{~dB})$. The tests were conducted on 8 subjects with normal hearing ( 5 women and 3 men), aged 22-37 years. Following statistical analysis it was found that: individual subjects' responses differed considerably with respect to angle of direction hearing acuity values, localization is most difficult at the angles of $90^{\circ}$ and $270^{\circ}$, worse localization for trams noise were stated, RC signals are better localized than TR signals.
\end{abstract}

PACS: 43.66.Qp

\section{Introduction}

Localization of sound is a basic function of the auditory system. In the day-to-day life the directional hearing is used almost all the time in monitoring environment. Every person is affected by propagating sounds present in the environment. Unlike the eyes, the ears are always open and irrespective of our state of consciousness we can always hear the sounds of the environment in its entire spatiality. We can hear sounds even when it is dark or foggy - this ability of locating sound sources in space combined with our sense of hearing can help us to find or to avoid objects. It is the hearing analyzer that plays a significant role in spatial orientation while other senses make important contribution to the perception [1].

Although the physiological mechanisms that operate in the process of sound localization are likely to be extremely complicated, a simplified conceptual structure as a representation of the major phenomena can be adopted. When this simplifying conception is adopted and one can assume that inputs to the brain are restricted to just two pressure-versus-time wave forms, the three basic cues are: the interaural time difference (ITD), interaural intensity difference (IID), and the monaural frequency spectrum of the received signal at each ear. There should be

* corresponding author; e-mail: niewiaro@ump.edu.pl noted some other factors that influence the localization of sound sources. One set of factors that may influence the judgements of the listeners is knowledge about the sound source and the acoustic characteristics of the surroundings. In addition, there are nonacoustic cues such as motion or position of the head and visual information that must be considered [2].

Mills was the first to attempt the solution of the quantitative problem connected with the determination of the spatial segregation of the auditory system [3]. He defined the audible angle, perceived by the subject as the just noticeable change of the angle from which the sound propagates with respect to the location of the reference sound source (minimum audible angle, MAA). At about the same time Zakrzewski developed a method, which also permits determination of angular values for the position of the sound source [4]. In his study the subject received two consecutive signals from different places within a circle around which the loudspeaker was moving (the subject was located in the centre). The smallest angle between two loudspeaker positions when the subject recognizes that both signals come from two different places in the space was called the angle of directional hearing acuity (ADHA).

The problem becomes more and more significant in present times when people are constantly exposed to acoustic stimuli, which are different with respect to their quality and intensity. In the environment, in which 
sounds are produced and where they propagate, each man is exposed to them, constantly hears the surrounding environment and to a larger or smaller extent is able to localize a given sound in space. The problem of localization becomes particularly important in the case of urban noises, mainly traffic noises (sound sources are mainly represented by moving objects), when the life of disabled people and particularly blind or visually impaired people, is at stake.

The appearance of acoustic emitters at pedestrian crosswalks (the sixties of the XX century) resulted in numerous studies which aimed to determine which signals are most applicable for this type of application. Hulscher found that, because of the masking of high frequency signals by predominantly low frequency traffic noise, and because a majority of blind pedestrians have some upper frequency hearing loss, the optimal fundamental frequency of the tone should be between $300 \mathrm{~Hz}$ and $1000 \mathrm{~Hz}$, and the tone should be comprised of multiple short bursts of sound to aid localization [5]. He also showed that the best perceived signal in the background of traffic noise is a signal of a frequency of $880 \mathrm{~Hz}$. Furthermore, this signal is also best absorbed by the windows, so it is least annoying to people living around the crosswalks [6]. Standards state that the sounds used in the acoustic emitters should not be ambiguous, that is should not be associated with any other information, should not resemble any known source of sound which could distort the information about the possibility of crossing the road. These signals should be both well-perceived by persons who are crossing the street and to provide a good localization of the sound emitter [7-9].

The aim of the study was to find a signal which would be optimal to use in the emitters at pedestrian crosswalks from the point of view of localization ability. The experiments were performed on signals, which provide good perception against the background noise even for people with hearing loss. There were also taken into account the environmental conditions associated with the assertion of a minimum signal annoyance for people living around the pedestrian crosswalks with sound emitters.

\section{Methodics}

The ADHA parameter was assumed as the measure which defines localization ability [4]. The study was conducted with a mobile loudspeaker system. The loudspeaker was fixed to an arm with a radius of $1.5 \mathrm{~m}$ and moved around a circle; the subject's head was positioned in the middle. The subject was seated in a chair with a special support, which prevented the subject from moving the head in an uncontrolled way [10]. The arm with the loudspeaker was positioned using a stepper motor. The motor was controlled by means of a controller programmed via a $\mathrm{PC}$ and Matlab.

ADHA measurements were made for 8 azimuths in the horizontal plane at the height of subject's head in the range of $0^{\circ}-360^{\circ}$, every $45^{\circ}$. The measurement was repeated with the listener's position changing clockwise.
At azimuths of $45^{\circ}, 90^{\circ}$ and $135^{\circ}$ the loudspeaker was positioned opposite to the listener's left ear, at azimuths $225^{\circ}, 270^{\circ}$ and $315^{\circ}$ — opposite to the listener's right ear. At angle $0^{\circ}$ the listener sat exactly in front of the loudspeaker and at angle $180^{\circ}$ — with his back to the loudspeaker (see Fig. 1).

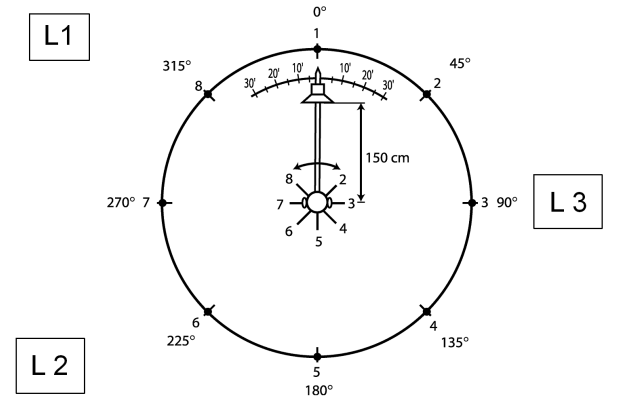

Fig. 1. The listener's position with respect to the loudspeaker emitting a given signal.

The two alternative forced choice (2AFC) method was used to determine the ADHA values. The loudspeaker generated 2 signals in a sequence, from 2 different positions. The value of the angle between successive positions of the loudspeaker in a single test was determined on the basis of the subject's responses and the rules of the adaptative procedure. After each pair of stimuli was generated, the subject was asked to say whether the second signal in the pair was to the right or to the left of the first signal $[11,12]$. In the experiment the $2 \mathrm{AFC}$ procedure with 6 turning points and an additive step was used: large additive step $4^{\circ}$ and small additive step $1^{\circ}$. A single threshold was determined as an arithmetic mean of the last 4 turning points.

At the first stage an analysis of the distribution of ADHA values was made. It was found that the distribution of ADHA values for different azimuths is asymmetric. For this reason, in the analysis of ADHA values a positional measure in the form of the median was adopted.

\section{Experimental investigations}

\subsection{Signals}

On the basis of psychoacoustic investigations (annoyance estimation, detection of threshold of signals presented in noise) conducted prior to the experiment [13], two signals were identified, which met the standard requirements for sound emitters:

- TR signal — a signal with a triangular temporal envelope and a sinusoidal carrier with a frequency of $880 \mathrm{~Hz}$, repeated periodically with a frequency of $5 \mathrm{~Hz}$,

- RC signal - a signal with a rectangular temporal envelope and a rectangular carrier with a basic frequency of $880 \mathrm{~Hz}$, repeated periodically with a frequency of $5 \mathrm{~Hz}$.

The signals were generated with the sampling frequency of $44100 \mathrm{~Hz}$ and the resolution of 16 bits. Time 
duration was $1.5 \mathrm{~s}$ and the interval between the signals — approximately $6 \mathrm{~s}$.

\subsection{Scenery}

Studies of ADHA in the background noises were carried out in the scenery, which schematically is shown in Fig. 1. The loudspeakers L1 and L2 emitted signals that simulate the noise generated by the moving sources (cars and trams) and the speaker L3 - by the non-moving sources (cars). The experiments were conducted for the following configurations of the background noise:

- non-moving cars and moving cars,

- non-moving cars and moving trams.

The averaged level of traffic noise in the experiments was $75 \mathrm{~dB}$ SPL and the averaged level of test signals $65 \mathrm{~dB}$ SPL $(\mathrm{SNR}=-10 \mathrm{~dB})$.

The tests were conducted on 8 subjects with normal hearing ( 5 women, 3 men), aged 22-37 years (average 26 years). The subjects listened to the signals in the anechoic chamber at the Institute of Acoustics, Adam Mickiewicz University which meets the requirements of ISO 3745-1977.

\section{Results and analysis}

Following a statistical analysis (ANOVA variance analysis) it was found that there are important differences between subjects $(F(7,512)=23.01 ; p<0.001)$, azimuths $(F(7,512)=88.492 ; p<0.001)$ and signals $(F(1,512)=$ 91.072; $p<0.001)$. On the other hand, there were no stated statistically significant differences depending on the type of the traffic noise $(F(1,512)=0.27 ; p=0.87)$. Figures 2 and 3 present ADHA results in the form of median, lower and upper quartile for all subjects but separately for each type of traffic noise and each type of test signal. The values of ADHA median for the sinusoidal signal with triangular envelope (TR) are higher than the value of ADHA median for a rectangular wave signal (RC). Such values were obtained for both the car noise (Fig. 2) and the tram noise (Fig. 3).

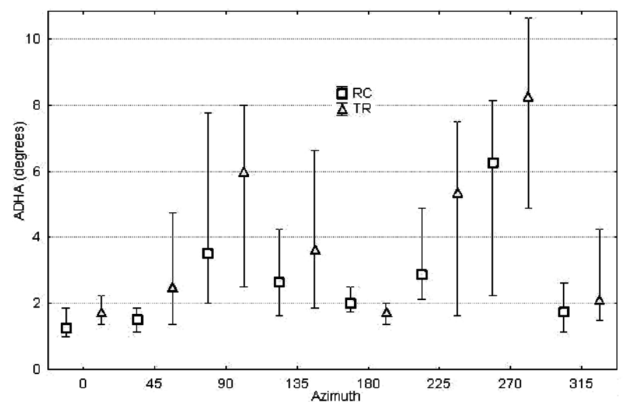

Fig. 2. ADHA median, lower and upper quartile obtained for the car noise for all subjects.

A post-hoc test was conducted for the subjects. Tukey's test enabled to identify the largest homogeneous group consisting of subjects no. $2,5,7,8$. The variance

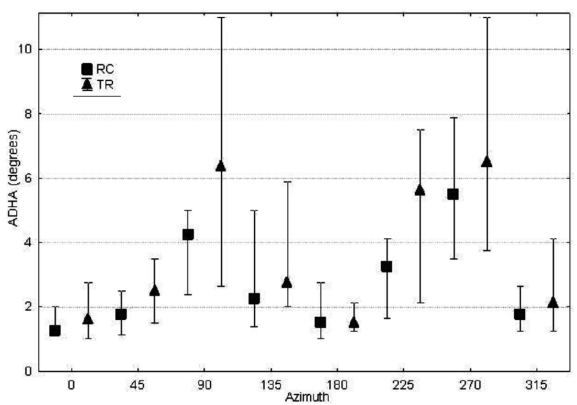

Fig. 3. ADHA median, lower and upper quartile obtained for the tram noise for all subjects.

analysis conducted for this group of subjects confirmed the absence of statistically significant differences between these four subjects $(F(3,256)=1.719 ; p=0.16)$. Figure 4 shows the values of ADHA obtained for the homogeneous group of subjects. The results were averaged over both the subjects and types of noise.

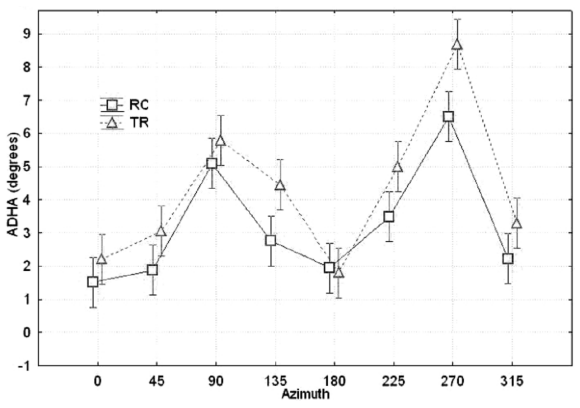

Fig. 4. ADHA values averaged over noise and homogeneous group of subjects.

It follows from the diagram in Fig. 4 that a much better localization is obtained for the RC signal.

\section{Conclusion}

The following conclusions can be drawn:

- there are considerable differences in ADHA values between individual subjects - they are clearly seen in the case of the TR signal, presented against the background of trams noise; the smallest differences were observed for the $\mathrm{RC}$ signal against the background of the same noise;

- a multifactorial variance analysis revealed that there are no significant differences in ADHA values for both types of the traffic noise;

- ADHA median values determined for all subjects are higher in the case of the TR signal, which indicates worse localization compared with RC signals;

- following the Tukey test, the largest homogeneous group was identified, consisting of 4 subjects; the 
variance analysis confirmed the absence of statistically significant differences between these subjects;

- ADHA values, averaged over noise and homogeneous group of subject, clearly indicate better localization of the RC signal compared to the TR signal.

\section{Acknowledgments}

The work was supported by the National Centre for Investigation and Development, grant N R11 000804.

\section{References}

[1] F.L Wightman, D.J. Kistler, in: Binaural and Spatial Hearing, Eds. R.H. Gilky, T.R. Anderson, LEA Publishers, Mahvah, New Jersey 1997.

[2] R. Hausler, S. Colburn, E. Marr, Acta Oto-Laryngol. 400, 1 (1983).

[3] A.W. Mills, J. Acoust. Soc. Am. 30, 237 (1958).
[4] A. Zakrzewski, Bull. Soc. Amis Sci. Lettr. Poznań, Serie c - Livraison X 9, 1 (1960).

[5] F. Hulscher, Australian Road Res. Board Proc. 8, 13 (1976).

[6] T. Poulsen, Appl. Acoust. 15, 363 (1982).

[7] List of Act No. 220-.2003, entry 2181 of 23.12 (annex no. 3 point 3.3.5) Decree of Ministry of Infrastructure, 2003.

[8] Polish Norm PN-Z-80100-2004 (2004).

[9] ISO 23600-2007(E), 2007.

[10] M. Niewiarowicz, J. Czajka, E. Hojan, Arch. Acoust. 30, 1 (2005).

[11] M. Niewiarowicz, in: Proc. "7th Conf. Acoustics in Audiology and Phoniatrics, Poznań, 2008, abstract.

[12] M. Niewiarowicz, A. Furmann, in: Proc. 20th Int. Congress on Acoustics, Sydney, Eds. M. Burgess, J. Davey, C. Don, T. McMinn, Australian Acoustical Society, Sydney 2010.

[13] E. Bogusz, A. Furmann, Acta Phys. Pol. A 119 (Supplement), 925 (2011). 